\title{
Scanning and Transmission Electron Microscopic Studies on Isolated Ruptures of Chordae Tendineae
}

\author{
Ying-Shiung LeE, M.D., F.C.C.P., * Chau-Hsiung Chang, M.D., \\ Hsin-Chin Chen, and Gan-Fang Liang
}

\section{Summary}

Electron microscopic studies of chordae tendineae of the mitral valve were carried out in 17 patients who underwent mitral valve replacement due to a spontaneously isolated rupture of chordae tendineae. The normal chordae, used as the control group, were obtained at autopsy from 5 patients who died from extracardiac causes and were compared with the ruptured chordae. In all patients with chordal rupture, scanning electron microscopy showed perforations of the chordae tendineae, with extensive desquamation and disruption of the endothelial cells and wide-spread destruction of the collagen fiber bundles in the central collagenous core. These pathological findings were not observed in the normal chordae from the control group. Transmission electron microscopy showed that the ruptured chordae were characterized by heterogeneous collagen fibrils with intrinsic structural alterations and disorganization in fibril arrangement. There was a wide variation in the diameters of collagen fibrils which always showed abnormal morphology, with abnormally large, peculiarly shaped fibrils. Apparent loss and/or a disordered arrangement of the typical periodicity of the fibrils were frequently observed. In addition, various degrees of degenerative changes of collagen tissue were often present. These abnormalities were never seen in the fibrils of the normal chordae, and were observed consistently in both the fibrils of the ruptured chordae and in the macroscopically intact chordae in the group with spontaneous rupture of chordae tendineae. These results suggest that a defective organization of collagen into fibrils and fibers, associated with secondary degeneration of collagen within the central collagenous core of the chordae tendineae, are important pathogenetic mechanisms for spontaneously isolated ruptures of chordae tendineae.

From the Cardiovascular Division, Department of Internal Medicine, Chang Gung Memorial Hospital, Taipei, Taiwan, R.O.C.

* Director in Cardiovascular Division.

Address for reprint: Ying-Shiung Lee, M.D., Chang Gung Memorial Hospital, 199, Tung Hwa North Road, Taipei, Taiwan, R.O.C.

Received for publication June 15, 1982. 


\section{Additional Indexing Words:}

Electron microscopy Chordae tendineae Ultrastructural heterogeneity

G PONTANEOUS rupture of chordae tendineae of the mitral valve, one $D$ of the major causes of isolated mitral regurgitation, has distinctive clinical features that justify its designation as a clinical syndrome. ${ }^{1)-5)}$ The most common form of ruptured chordae is an idiopathic form of unknown etiology. ${ }^{2}$ Although it is considered by some investigators as part of the general concept of myxomatous degeneration of the mitral valve, it is still a matter of debate in the literature. ${ }^{2), 3)}$ Since collagen is the major structural component of the chordae tendineae which are under continuous, repetitive mechanical stress, alterations in collagen, even at the molecular level, might be expected to lead to the weakening or disruption of the chordae tendineae. To date, relatively little is known of the role of collagen abnormalities in the pathogenesis of isolated ruptures of the mitral chordae tendineae. Therefore, the purpose of the present report is to describe the results of a combined study, which uses scanning and transmission electron microscopy to investigate collagen defects in the idiopathic form of ruptured chordae tendineae, with particular emphasis on the morphological characteristics and intrinsic structural alterations of collagen fibrils.

\section{Materials and Methods}

This study analyzed specimens from 17 patients who underwent mitral valve replacement due to a spontaneous rupture of chordae tendineae with neither apparent heart disease nor a history of chest injury. The patients included 13 men and 4 women, ranging in age from 17 to 68 years. The diagnosis of an idiopathic form of isolated rupture of the chordae tendineae was confirmed by operative and pathologic findings. We excluded the patients with chordal rupture due to bacterial endocarditis of various etiologies and of valvular heart disease (including a floppy mitral valve), ${ }^{6), 71}$

Immediately after the excision of the mitral valves during open heart surgery, both the ruptured and the macroscopically intact chordae were resected separately. Normal chordae, used as the control for comparative study, were obtained at necropsy from 5 patients who died from extracardiac causes. In each case, we obtained at least 10 chordae, which were trimmed and fixed with ice-cold $3 \%$ glutaraldehyde in $0.1 \mathrm{M}$ phosphate buffer $(\mathrm{pH}$ 7.2-7.4) for 2 hours. Subsequently the specimens were rinsed in several changes of cold phosphate buffer and postfixed in $2 \%$ phosphate-buffered 
osmium tetroxide ( $\mathrm{pH}$ 7.2-7.4) for an additional 2 hours. After fixation the tissues were again rinsed in $0.1 \mathrm{M}$ phosphate buffer and dehydrated in graded concentrations of chilled ethanol.

For scanning electron microscopy (SEM) the dehydrated tissues were subjected to critical point drying. The samples were then placed in liquid nitrogen, fractured with a pre-cooled razor blade, mounted on specimen stubs for SEM, and coated with gold. Then the specimens were examined with a Hitachi H-5010 SEM, operated at $25 \mathrm{KV}$. For transmission electron microscopy (TEM) the dehydrated tissues were embedded in Epon-Araldite. The specimens were sectioncd with a Sorvall MT-2B Porter-Blum ultramicrotome using glass knives and stained with uranyl acetate and lead citrate. The ultrathin sections were examined with a Hitachi H-500 electron microscope, operated at $75 \mathrm{KV}$.

For comparison of the diameters of the collagen fibrils, we arbitrarily measured two-hundred fibrils in cross section at high magnification $(\times 200,000)$ from both the normal control specimens and the specimens from patients with chordal rupture. Data were analysed using Student's t-test. A significant difference between groups was defined as a $p$ value less than 0.05. In addition, we counted 580 randomly selected fibrils from five electron-micrographic images at high magnification $(\times 100,000)$ to estimate the percentage of different types of collagen fibrils in the group with ruptured chordae.

\section{Results}

\section{Scanning electron microscopic (SEM) findings}

The morphological features of the chordae tendineae from the superficial endothelial surface to the central collagenous core (Pars fibrosa) could be visualized in all specimens studied with SEM. The normal chordae had a dense central mass of collagen covered by intact endothelium, which occasionally showed sloughing off of the individual endothelial cells. By contrast, perforated holes on the endothelial surface (Fig. 1), with extensive desquamation and disruption of the endothelial cells (Fig. 2), were always observed in the group of ruptured chordae tendineae. The individual endothelial cells were usually covered with numerous microvilli. In all cases examined, a number of irregular breaks of varying sizes were often present in the central collagenous core (Fig. 3). The collagen fiber bundles in these affected areas were randomly arranged with extensive disruption (Fig. 4). These pathological changes were noticed not only in the ruptured chordae but also in the macroscopically intact chordae. 


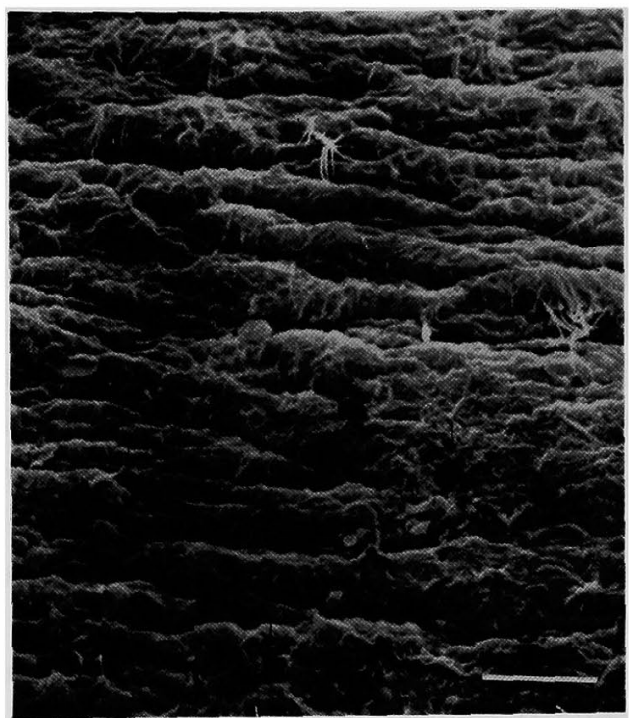

Fig. 1. Scanning electron micrograph of the pcrforated holes (arrows) on the endothelial surfaces of the ruptured chordae tendineae. Horizontal bar $=10 \mu(\times 1,700)$.

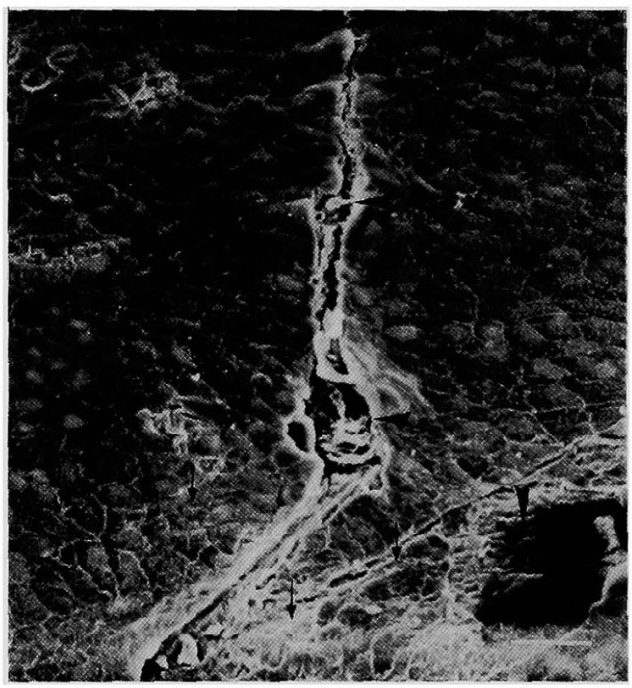

Fig. 2. Scanning electron micrograph of the endothelium showing desquamation of the endothelial cells (arrows) and irregular disruption and perforation of the endothelium (arrow heads). Horizontal bar $=50 \mu(\times 280)$.

\section{Transmission electron microscopic findings}

I. Heterogeneous collagen fibrils with intrinsic structural alterations

The collagen fibrils in the normal control group had cylindrical shapes with mild variations in the diameter (Fig. 5), ranging from 42.5 to $57.5 \mathrm{~nm}$, 

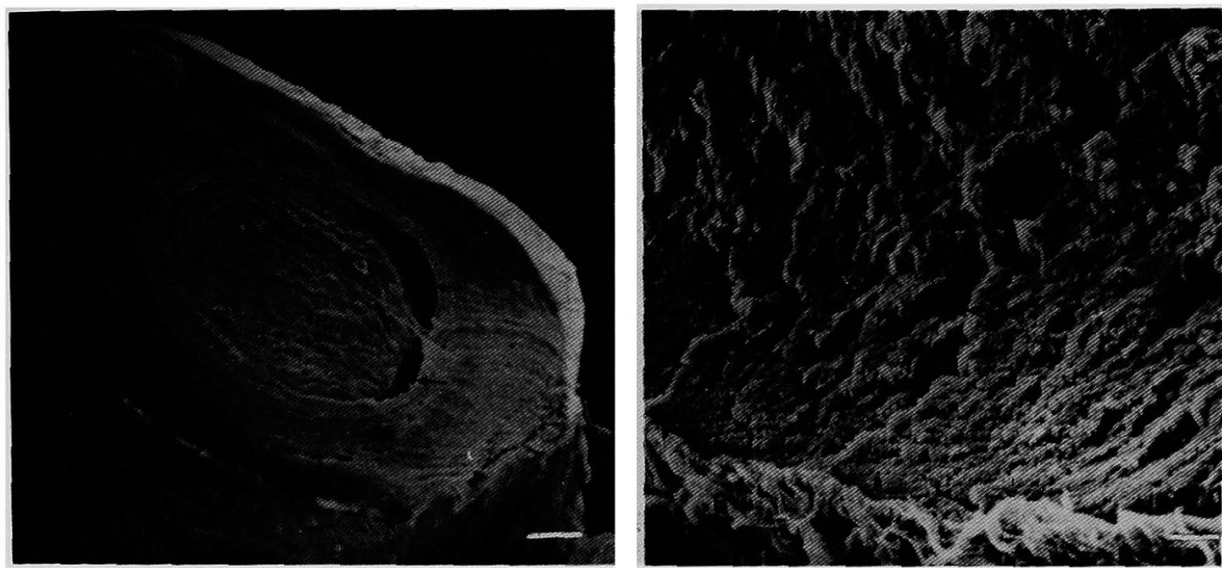

Fig. 3. Scanning electron micrographs of the ruptured chordae in cross section showing irregular breaks in the central collagenous core (arrows). A (left), Horizontal bar $=100 \mu(\times 100)$; B (right), Horizontal bar $=10 \mu(\times 860)$.

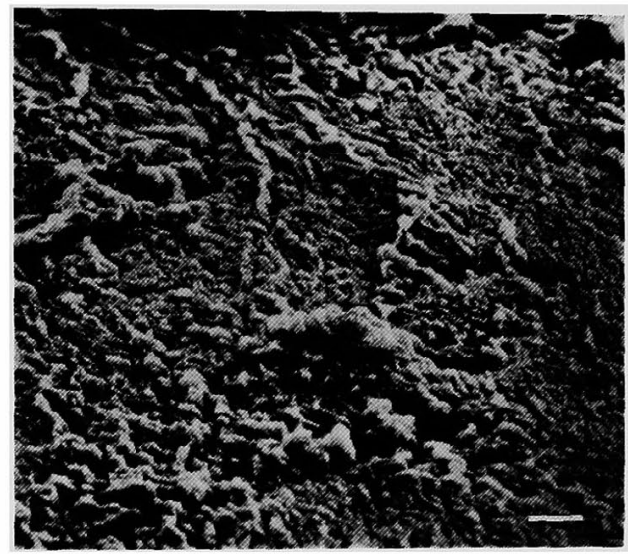

Fig. 4. Scanning electron micrograph of the collagen fiber bundles in the pars fibrosa showing disorganized arrangement and extensive disruption. Horizontal bar $=10 \mu(\times 860)$.

with a mean of $48.33 \mathrm{~nm}$ (S. D. $\pm 3.02 \mathrm{~nm}$ ). The most characteristic finding in the ruptured chordae tendineae was the abnormal morphology of the collagen fibrils, which varied widely in diameter from 20.0 to $150.0 \mathrm{~nm}$ (Fig. 6). It was possible to unambiguously classify the collagen fibrils into three different categories on the basis of fibril diameters and morphological features. Type A fibrils had diameters larger than the maximum diameter $(57.5 \mathrm{~nm})$ of the collagen fibrils in the control group, varying from 60.0 to $150.0 \mathrm{~nm}$, with a mean of $90.27 \mathrm{~nm}$ (S. D. $\pm 24.13 \mathrm{~nm}$ ). The most characteristic feature of this type was a markedly irregular shape which often showed finger-printed appearance (Fig. 7). Type B fibrils were almost identical with those seen in 

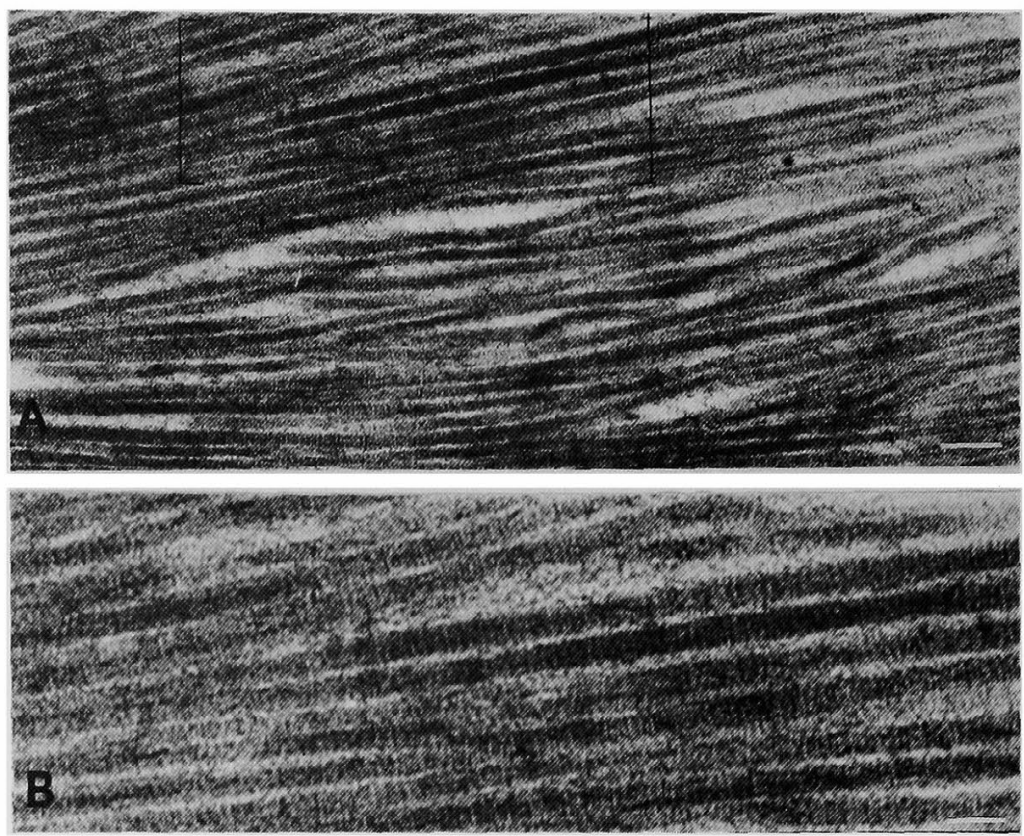

Fig. 5. Electron micrographs of collagen fibrils in the normal chordae tendineae of the control group showing almost uniform diameters. The collagen fibrils are densely packed in an orderly, parallel array (A). Higher magnification of the bracket in A shows the characteristic periodicity in the fibrils (B). A, Horizontal bar $=200 \mathrm{~nm}(\times 50,000)$; B, Horizontal bar $=$ $100 \mathrm{~nm}(\times 100,000)$.

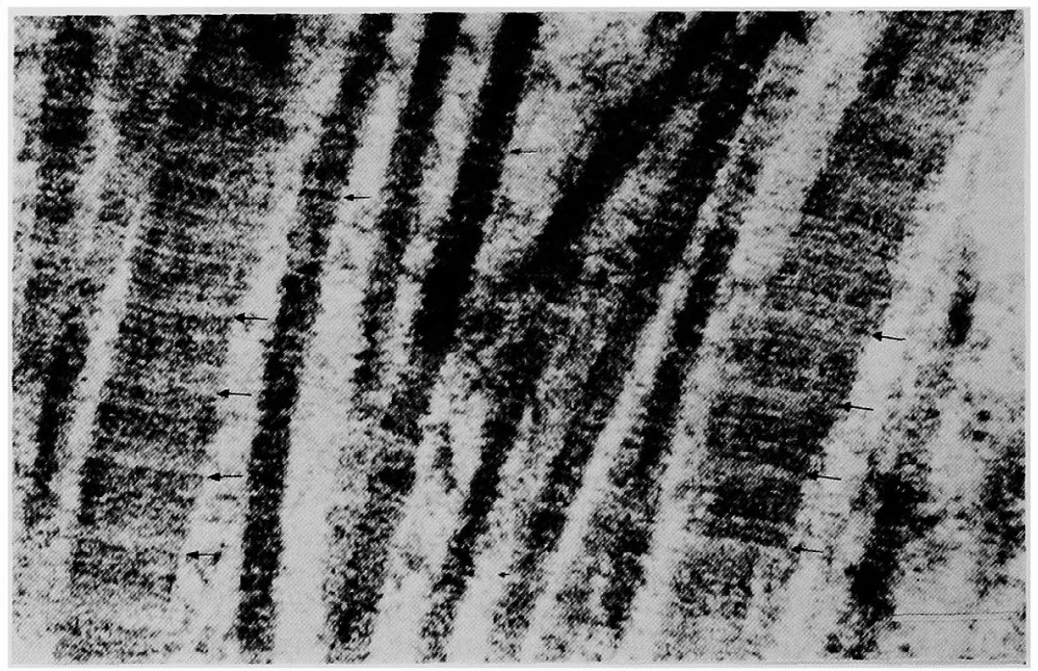

Fig. 6. Electron micrograph of collagen fibrils in the ruptured chordae tendineae showing a wide variation in fibril diameters. Note the sizes of two abnormally large fibrils (large arrows) are nearly three times larger than the normal ones (small arrows). The characteristic periodicity in the fibrils is still preserved but seems deranged. Horizontal bar $=100 \mathrm{~nm}(\times 200,000)$. 


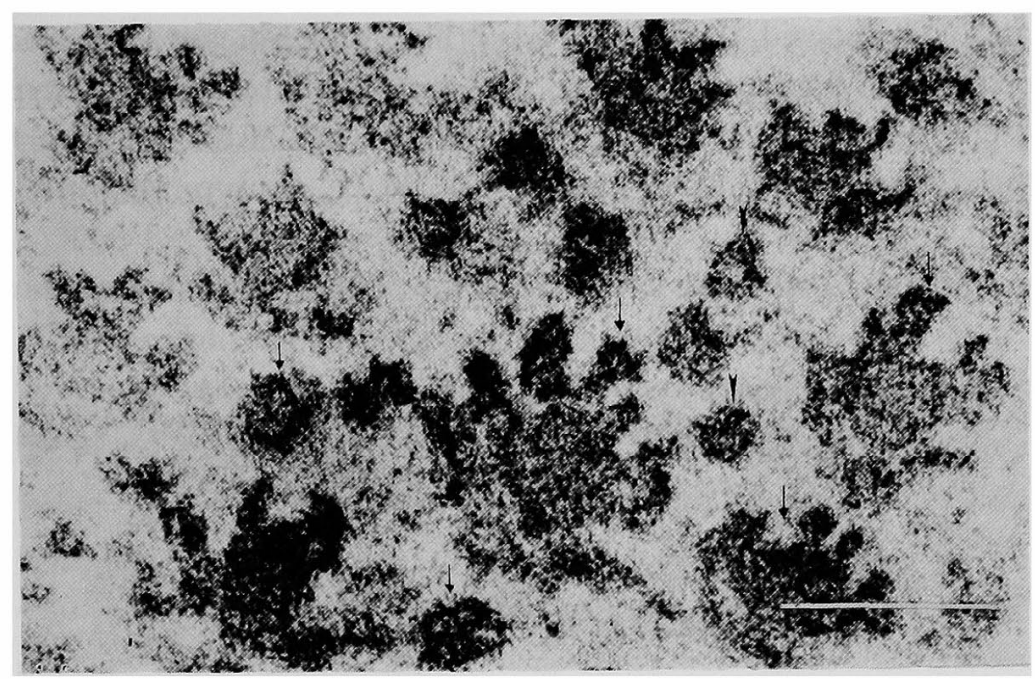

Fig. 7. Electron micrograph of various types of collagen fibrils in the ruptured chordae. Note the finger-printed appearance of the type A fibrils (large arrows). The type $\mathrm{B}$ and $\mathrm{C}$ fibrils are indicated by small arrows and arrows heads respectively. Horizontal bar $=100 \mathrm{~nm}(\times 360,000)$.

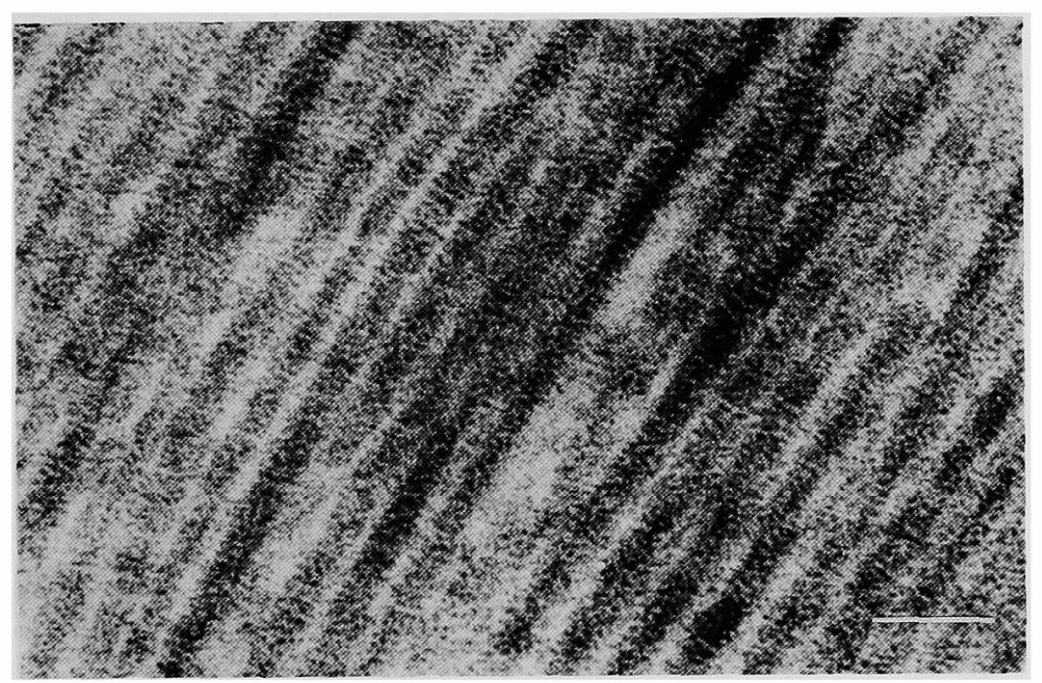

Fig. 8. Electron micrograph of collagen fibrils in the ruptured chordae showing apparent loss of the characteristic periodicity in the fibrils. Horizontal bar $=100 \mathrm{~nm}(\times 200,000)$.

the control group. The fibril diameters were between 45.0 and $57.0 \mathrm{~nm}$, with a mean of $52.01 \mathrm{~nm}(\mathrm{~S} . \mathrm{D} . \pm 3.41 \mathrm{~nm})$. Type C fibrils showed diameters less than the smallest diameter $(42.5 \mathrm{~nm})$ of the fibrils in the control group, ranging from 20.0 to $40.0 \mathrm{~nm}$, with a mean of $31.41 \mathrm{~nm}$ (S. D. $\pm 4.64 \mathrm{~nm}$ ). In general, these small fibrils still maintained a cylindrical shape. The sta- 
tistical analyses showed that there were highly significant differences $(p<$ 0.001 ) in the fibril diameters between the normal control and each type of the ruptured chordae. The percentage of various types of the collagen fibrils in the ruptured chordae was $31.9 \%$ for type A, $26.7 \%$ for type B, and $41.4 \%$ for type C.

The collagen fibrils were normally characterized by periodic staining pat-

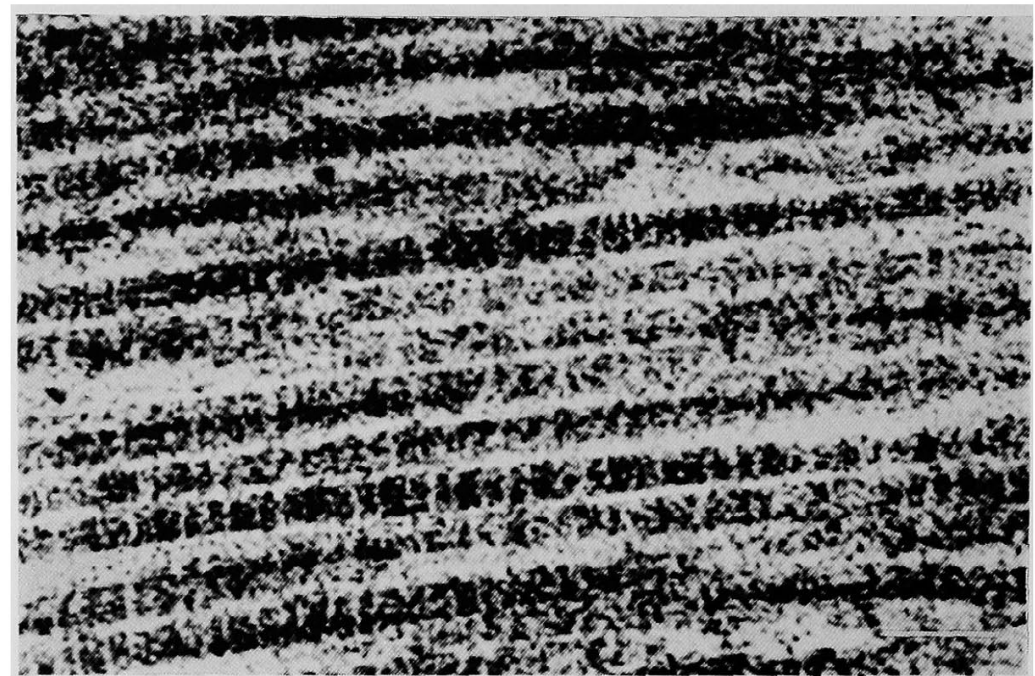

Fig. 9. Electron micrograph of collagen fibrils in the ruptured chordae showing disorganization of periodic banding pattern. Horizontal bar = $100 \mathrm{~nm}(\times 200,000)$.

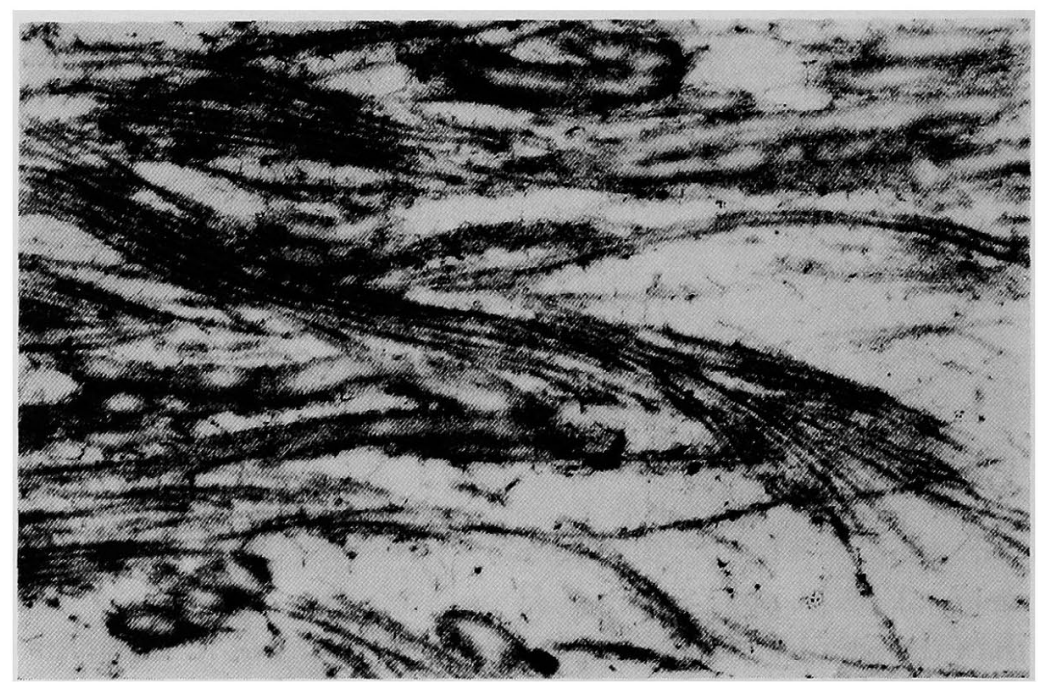

Fig. 10. Electron micrograph of collagen fibrils in the ruptured chordac showing a very loosely packed and disorganized arrangement. $\times 35,000$. 
terns, varying from 60 to $70 \mathrm{~nm}$ (Fig. 5). The most obvious change in the intrinsic structure of collagen fibrils in the ruptured chordae was an apparent loss of typical periodicity (Fig. 8). The disordered arrangement of the periodic banding pattern in the fibrils was another characteristic, frequent finding (Fig. 9). The heterogeneity in the morphology of the collagen fibrils with intrinsic structural alterations was observed consistently in both the ruptured chordae and the macroscopically intact chordae in specimens from patients with a spontaneous rupture of chordae tendineae. However, these abnormal findings were never seen in the fibrils of the control group.

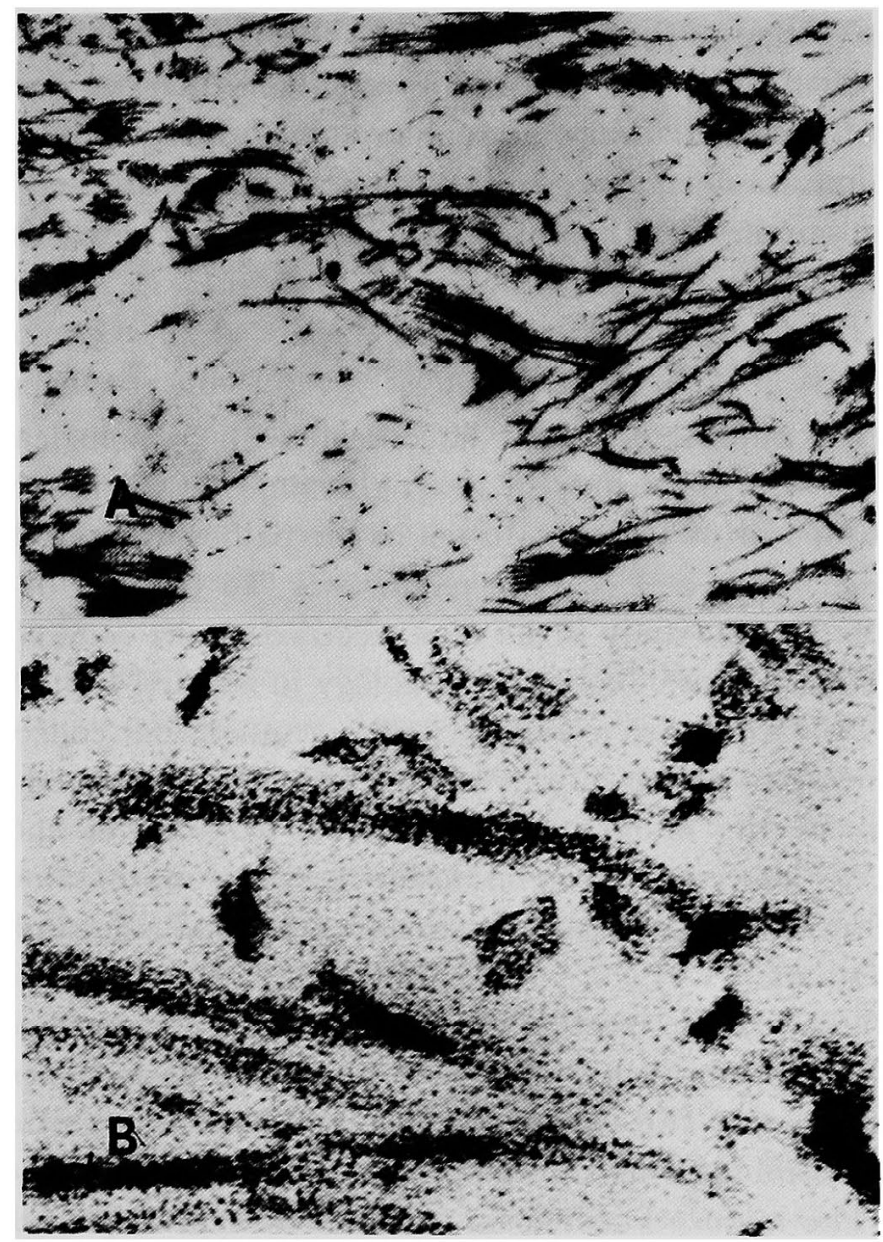

Fig. 11. Electron micrographs of the ruptured chordae showing extensive loss of collagen tissue with the individual collagen fibrils fragmented and coiled. A number of particles are observed intimately associated with the fragments of the fibrils (B). Note a loss and/or disorganization of periodicity in the fragments of collagen fibrils (B). A, $\times 20,000 ; B, \times 200,000$. 
II. Degenerative changes in collagen tissue

The collagen fibrils were closely packed with a fairly parallel arrangement in the control samples (Fig. 5). In samples with ruptured chordae the collagen fibrils were less densely packed and more irregularly arranged (Fig. 10). Large areas showed an apparent loss of collagen tissue, with the individual fibrils fragmented, coiled, and disrupted (Fig. 11). A great number of particles, nearly equal in size to the fibrils, were often noticed around or intimately associated with the fragments of fibrils. These pathological findings were quite compatible with scanning electron microscopic observations which revealed disorganization and disruption of the fiber bundles. These were no demonstrable pools of amorphous ground substance in areas where destruction of collagen tissue was present. By contrast, areas with severe degenerative changes of collagen usually showed a marked decrease or loss of ground materials containing residual strands of collagen. In general, we observed a similar amorphous ground substance in less affected areas of collagen tissue in samples with ruptured chordae as in the control tissue.

\section{Discussion}

Although a number of studies have dealt with pathological changes in the cardiac valves in a variety of heart disease, ${ }^{6)-11)}$ essentially nothing is known about the role of altered collagen in the pathogenesis of the idiopathic form of isolated rupture of chordae tendineae. To the best of the authors' knowledge, the present study is the most detailed report concerning electron microscopic observations of collagen pathology in isolated rupture of chordae tendineae. To date, much confusion still surrounds the concept of myxomatous degeneration of the mitral valve, which was considered by some investigators to be the etiology of spontaneous rupture of chordae tendineae. ${ }^{2,3), 11)-14)}$ However, the relationship between myxomatous degeneration and ruptured chordae tendineae remains a significant and largely unanswered question.

Since collagen is the most abundant and major structural component of the chordae tendineae, the function of the chordae clearly depends on the integrity of collagen. The collagen fibrils, the final product of collagen biosynthcsis, arc asscmbled from the completed collagen through a serics of reactions. ${ }^{15)-19}$ The mature fibrils, characterized by typical periodicity have a cylindrical shape and an almost uniform diameter, which is the result of a precise aggregation of collagen molecules and perfect control of the lateral growth of collagen fibrils. ${ }^{201-23)}$ Aberrations in the biosynthetic pathways of collagen may lead to pathological defects in collagen fibrils and fibers caus- 
ing malfunctions of chordae tendineae which will have clinical ramifications. At least three populations of polymorphic forms of collagen fibrils, with peculiar shapes and variable diameters, and an abnormal proportion of morphologically normal and abnormal fibrils were the most characteristic observations associated with an isolated rupture of the chordae tendineae. We suggest that the morphological heterogeneity of collagen fibrils in the ruptured chordae results from a defect in the packing of individual collagen molecules into fibrils or a loss of control of the lateral growth of the fibrils. In addition, a less organized arrangement of collagen fibrils further indicates a failure in the parallel packing of the collagen fibrils into fibers. Nothing is known about the mechanisms controlling growth and development of collagen fibrils, although noncollagen matrix components may play a significant role in the control of fibril growth and the packing of collagen fibrils into fibers. ${ }^{22), 23)}$ It is obvious that further investigations are necessary to clarify the relationship between the growth of collagen fibrils and noncollagenous matrix.

Collagen fibrils are characterized by a $68 \mathrm{~nm}(60-70 \mathrm{~nm})$ banded staining pattern, which serves to distinguish collagen fibrils from other extracellular fibrillar structures and to identify fragments of collagen fibrils. ${ }^{20)-22)}$ This typical periodicity results from a regular alignment of the alternating polar and nonpolar regions of amino acids in staggered collagen molecules within fibrils. ${ }^{24), 25)}$ Electron microscopic examination of collagen fibrils in the ruptured chordae showed disorganization and/or loss of periodicity in the fibrils. These features were never seen in the control group. Thus, a defect in the packing of collagen molecules into fibrils was suggested by our findings of intrafibrillar abnormalities. Since abnormal fibrils are generally degraded more rapidly than normal ones, degenerative changes in collagen may occur, leading to disruption of the pars fibrosa and, finally, to chordal rupture. Although some ultrastructural changes in the floppy mitral valve syndrome ${ }^{\text {7),8) }}$ are similar to those reported in our present study of the ruptured chordae, the unique feature of the heterogeneity for fibril morphology in isolated rupture of chordae tendineae was never described in floppy mitral valves. Therefore, we consider that an isolated rupture of chordae tendineae is the result of a specific disease process which is different from the floppy mitral valve.

In summary, the most characteristic findings in isolated rupture of mitral chordae tendineae were the heterogeneity in morphology of collagen fibrils with intrinsic structural alterations and the malalignment of the fibrils with disruption and fragmentation. We suggest that the abnormal fibrils with a disordered arrangement may result from the defective organization of collagen into fibrils and a failure in the controlling mechanisms at the level of fibril growth and fiber bundle formation. We conclude that the altered 
collagen fibrils, with secondary degeneration of collagen within the central core of the chordae, may play an important role in the pathogenetic mechanisms of the spontaneous, isolated rupture of chordae tendineae. Further investigations are needed on the relationship of particular biochemical defects in collagen biosynthesis to this particular disorder.

\section{ReFERENCES}

1. Phillips HR, Levine FH, Carter JE, Boucher CA, Osbakken MD, Okada RD, Akins CW, Daggett WM, Buckley MJ, Pohost GM: Mitral valve replacement for isolated mitral regurgitation. Analysis of clinical course and late postoperative left ventricular ejection fraction. Am J Cardiol 48: 647, 1981

2. Selzer A: Nonrheumatic mitral regurgitation. Mod Concepts Cardiovasc Dis 48: 25, 1979

3. Selzer A, Katagama F: Mitral regurgitation. Clinical patterns, pathophysiology, natural history. Medicine 51: 337, 1972

4. Selzer A, Kelly JJ Jr, Vannitamby A, Walker P, Gerbode F, Kerth WJ: The syndrome of mitral insufficiency due to isolated rupture of the chordae tendineae. Am J Med 43: 822, 1967

5. Sweatman T, Selzer A, Kamagaki M, Cohn K: Echocardiographic diagnosis of mitral regurgitation due to ruptured chordae tendineae. Circulation $\mathbf{4 6}: 580,1972$

6. Frable WJ: Mucinous degeneration of the cardiac valves-the "floppy valve syndrome ". J Thorac Cardiovasc Surg 58: 62, 1969

7. Olsen EGJ, Al-Rufaie HK: The floppy mitral valve. Study on pathogenesis. Br Heart J 44: 674,1980

8. Davies MJ, Moore PB, Braimbridge MV: The floppy mitral valve. Study of incidence, pathology and complications in surgical, necropsy and forensic material. Br Heart $\mathrm{J} 40$ : 468,1978

9. Kern WH, Tucker BL: Myxoid changes in cardiac valves. Pathological, clinical and ultrastructural studies. Am Heart J 84: 294, 1972

10. Pomerance A: Aging changes in human heart valves. Br Heart J 29: 222, 1967

11. Rippe J, Fishbein MG, Carabello B, Angoff G, Sloss L, Collins JJ Jr, Alpert JS: Primary myxomatous degeneration of cardiac valves. Clinical, pathological, haemodynamic and echocardiographic profile. Br Heart J 44: 621, 1980

12. Marshall CE, Shappell SD: Sudden death and the ballooning posterior leaflet syndrome. Arch Pathol 98: 134, 1974

13. McCarthy LJ, Wolf PL: Mucoid degeneration of heart valves: "blue valve syndrome". Am J Clin Pathol 54: 852, 1970

14. Torii S, Bashey RI, Nakao K: Acid mucopolysaccharide composition of human heart valve. Biochim Biophys Acta 101: 285, 1965

15. Bailey A J, Robbins SP, Balian G: Biological significance of the intermolecular crossinks of collagen. Nature 251 : 105, 1974

16. Bornstein P: The biosynthesis of collagen. Annu Rev Biochem 43: 567, 1974

17. Fessler JH, Fessler LI: Biosynthesis of procollagen. Annu Rev Biochem 47: 129, 1978

18. Fujii K, Tanzer ML, Cooke PH: Collagen fibrogenesis and the formation of complex crosslinks. J Mol Biol 106: 223, 1976

19. Tanzer ML: Cross-linking of collagen. Science 180: 561,1973

20. Fietzek PP, Kühn K: Information contained in the amino acid sequence of the $\alpha 1$ (I)-chain of collagen and its consequences upon the formation of the triple helix, of fibrils and crosslinks. Mol Cell Biochem 8; 141, 1975

21. Fietzek PP, Kühn K: The primary structure of collagen. Int Rev Connect Tissue Res 7 : 
1,1976

22. Minor RR: Collagen metabolism. A comparison of diseases of collagen and diseases affecting collagen. Am J Pathol 98: 227, 1980

23. Patterson DF, Minor RR: Hereditary fragility and hyperextensibility of the skin of cats. A defect in collagen fibrillogenesis. Lab Invest 37: 170, 1977

24. Bruns RR, Gross J: Band pattern of the segment-long-spacing form of collagen. Its use in the analysis of primary structure. Biochemistry 12:808, 1973

25. Wiedemann H, Chung E, Fujii T, Miller EJ, Kühn K: Comparative electronmicroscope studies on type-III and type-I collagens. Eur J Biochem 51:363, 1975 